\title{
Commentary: Transformation of Taxol-Stabilized Microtubules into Inverted Tubulin Tubules Triggered by a Tubulin Conformation Switch
}

\author{
Radhika J. Poojari* \\ Department of Biosciences and Bioengineering, Indian Institute of Technology Bombay, Mumbai, India
}

Keywords: biomaterials, hierarchical nanostructures, microtubules geometry, nanoscale delivery, reverse conformation, spermine

\section{A Commentary on}

Transformation of Taxol-Stabilized Microtubules into Inverted Tubulin Tubules Triggered by a Tubulin Conformation Switch

by Ojeda-Lopez, M. A., Needleman, D. J., Song, C., Ginsburg, A., Kohl, P. A., Li, Y., et al. (2014). Nat. Mater. 13, 195-203. doi:10.1038/nmat3858

OPEN ACCESS

Edited by:

Malcolm Xing,

University of Manitoba, Canada

Reviewed by:

Xiaozhong Qiu,

Southern Medical University, China Chuanglong $\mathrm{He}$,

Donghua University, China

${ }^{*}$ Correspondence:

Radhika J. Poojari drradhikapoojari@gmail.com

Specialty section: This article was submitted to Biomaterials,

a section of the journal

Frontiers in Materials

Received: 21 September 2016 Accepted: 07 December 2016 Published: 23 December 2016

Citation:

Poojari RJ (2016) Commentary: Transformation of Taxol-Stabilized Microtubules into Inverted Tubulin

Tubules Triggered by a Tubulin

Conformation Switch.

Front. Mater. 3:57.

doi: 10.3389/fmats.2016.00057
Tubulins represent the backbone of a living system. Every cell exists as a complex nanoscale machinery, specifically the cytoskeleton network of microtubules. These building blocks are composed of long, very rigid, and hollow charged biopolymers of $\alpha$ - and $\beta$-tubulin heterodimer protein subunits. These dynamic cylindrical constructs perform a myriad of cellular functions such as intracellular transport, cell motility, cell shape, and chromosome segregation during mitosis and act as tracks for the kinesin motor proteins (Bertalan et al., 2014; Katsuki et al., 2014). The tubulin dimers form linear protofilaments and assemble into a cylindrical structure with $\sim 25 \mathrm{~nm}$ outer diameter and $\sim 15 \mathrm{~nm}$ inner diameter. The length of microtubules can be $\sim 50 \mu \mathrm{m}$ to several micrometers and are negatively charged. Microtubules are dynamically unstable tubulin polymers in equilibrium with monomers that interconvert stochastically between growing and shrinking phases, vital for cellular functions. This rapid transition phase is referred to as the "turning on-off microtubules" phenomenon. The conformational switch is linked to the GTP hydrolysis in $\beta$-tubulin. The protofilaments are in straight conformation during polymerization of microtubules and in curved shape during depolymerization of microtubules (Bertalan et al., 2014; Katsuki et al., 2014). Due to the vital role of microtubules in mitosis, they are excellent anticancer drug targets such as Taxol and vinca alkaloids. The rationale of this commentary is based on the unique shape-remodeling supernanostructures of microtubules, which can be used as novel biomaterials for delivery of nanomedicines.

Microtubule bundles possess a tightly packed geometry. Nanoscale superstructures are the sources of inspiration for the design of complex materials, i.e., shape and surface pattern with advanced functionalities (Gröschel et al., 2013). By modifying the diameter of microtubules, one can control the nanotubes geometric parameters important in biomaterials applications. The basic geometry is the shape of nanoparticles, which influences these parameters tremendously with different aspect ratios. For example, nanoscale cylindrical particles with higher aspect ratios (diameter of $150 \mathrm{~nm}$ and height of $450 \mathrm{~nm}$ ) internalized rapidly (5.2\% of cells/min) into the cervical cancer HeLa cells in comparison to the symmetrical particles with lower aspect ratios (diameter of $200 \mathrm{~nm}$ and height of $200 \mathrm{~nm})$ at a lower rate $(1.2 \%$ of cells/min) as reported by Gratton et al. (2008). The cellular uptake depends on a shape of a nanoparticle and ultimately the designing of the nanoparticle for effective nanomedicine delivery (Chun, 2008; Brazil, 2016). Nogales et al. (2003) had reported 
tubulin ring structures in presence of divalent cations having stabilizing effects. The ring curvature is due to a kink between dimers and a kink at monomer-monomer interface of similar or decreased magnitude. Cheng and Stevens (2014) studied the selfassembly of tubular structures from macromolecular monomers with multiple binding sites on their surfaces and lock-and-key interactions to obtain controlled, self-assembled tubule pitch (strength of vertical interaction $>$ lateral interaction strength in microtubules). These unique properties of microtubules can be applied in nanoscience to create supramolecular systems. A monomer is a single nanoparticle with self-assembled tubular structures mimicking microtubules.

Materials scientists are motivated by technological advancements to alter or tailor the material of interest. This process has lead to discovery of a new class of functional materials and superior functionality as evidenced by a novel concept in the field of nanotechnology and microtubule dynamics. This commentary is based on the work published in Nature Materials in 2014 by Ojeda-Lopez et al. (2014) from University of California and other institutions who unveiled a remarkable change in the architecture of microtubule cytoskeletal assembly called as the inverted tubulin tubules. This conformational switch was induced by a polyamine spermine with $4+$ cationic charge. The transition from bundles of Taxol-stabilized microtubules $(26 \mathrm{~nm})$ to inverted tubulin tubules bundled phase $(40.4 \mathrm{~nm})$ was detected by transmission electron microscopy and synchrotron small-angle X-ray scattering (SAXS). These compact polymeric assemblies resembled like a stack of rings with the inside-out model system, wherein the outer surface of inverted tubulin tubules corresponds to the inner lumen of microtubules (Figure 1). The different aspects of hierarchically ordered nanostructures, electrostatic interactions, conformational transition, and nanoparticle assemblies have been elucidated. Experimental results have shown that the microtubules lateral and vertical surfaces cause the transition to form inverted tubulin tubule structures. SAXS data have shown microtubules bundled at $15 \mathrm{mM}$ spermine concentration, whereas, at $30 \mathrm{mM}$, it was completely in the inverted tubulin tubule bundled phase at room temperature after 10 days. This suggested that the ring formation leading to such inverted structures are induced by highly charged spermine localized in small volume between the lateral contacts in microtubule. Spermine lowers the straight-to-curved energy barrier with increasing concentrations. In addition, at $2.5 \mathrm{mM}$ constant spermine concentration at $\sim 2.5^{\circ} \mathrm{C}$, the transformation to inverted phase occurred rapidly after $12 \mathrm{~h}$ in contrast to stable microtubule bundled phase for $>10$ days at room temperature (Ojeda-Lopez et al., 2014). The structural properties of microtubules are crucial for nanotechnological applications. Needleman et al. (2004) had reported higher-order, nanometer scale microtubule assemblies in the presence of large trivalent, tetravalent, and pentavalent counterions form nanowires, sieves, tightly packed hexagonal bundles due to the large internal volume. Whereas, in the presence of small

\section{REFERENCES}

Bertalan, Z., La Porta, C. A., Maiato, H., and Zapperi, S. (2014). Conformational mechanism for the stability of microtubule-kinetochore attachments. Biophys. J. 107, 289-300. doi:10.1016/j.bpj.2014.06.004

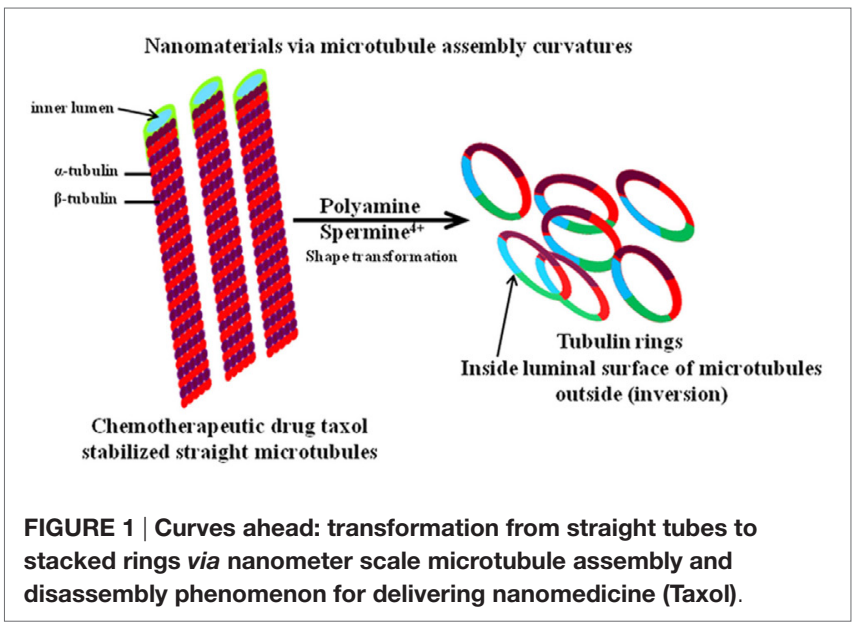

divalent cations, highly anisotropic living necklace bundles were formed due to the large surface area. Thus, microtubule bundling architectures of controlled dimensions as templates can be tailored for miniaturized materials. From the technological viewpoint, these nanomaterials find interesting applications in microtubulebinding drug, MAP-tau protein, dynamin protein, tubulin GTPase enzyme (Safinya et al., 2011; Ojeda-Lopez et al., 2014), and DNA gene (Koltover et al., 1998) encapsulation systems in the inside luminal surface of microtubules and release on triggered disassembly. They also find applicability in circuitry components such as carbon nanotubes and conducting polymers, thermoresponsive polymer (Thess et al., 1996; Kaiser, 2001; Needleman et al., 2004; Schroeder et al., 2013), in hierarchical nanostructures templates such as liposomes-microtubule complexes (Raviv et al., 2005; Safinya et al., 2011), and in biosensing devices (Malcos and Hancock, 2011).

In conclusion, microtubules represent an ideal biopolymer for investigating the nanoscale superstructures. The work by OjedaLopez and colleagues represents a new strategy for nanoinspired structures with well-defined shape and reversed surfaces (i.e., inside luminal surface of microtubules) via a small molecule, spermine triggered conformation switch in comparison to the conventional straight polymeric microtubule structures. The assembly and disassembly of microtubules forms a novel, solid platform for supernanostructures with on-demand triggered release of encapsulated biomolecules and distinct functions. The quest for innovative biomaterials like microtubules is a unique advancement in nanoscience and nanotechnology.

\section{AUTHOR CONTRIBUTIONS}

The author contributed in conceptualization and writing of this commentary.

Brazil, R. (2016). Shaping up at the nanoscale. Chemis. World 13, 62-65.

Cheng, S., and Stevens, M. J. (2014). Self-assembly of chiral tubules. Soft Matter 10, 510-518. doi:10.1039/c3sm52631c

Chun, A. L. (2008). Nanoparticles: shape also matters. Nat. Nanotechnol. doi:10.1038/nnano.2008.265 
Gratton, S. E., Ropp, P. A., Pohlhaus, P. D., Luft, J. C., Madden, V. J., Napier, M. E., et al. (2008). The effect of particle design on cellular internalization pathways. Proc. Natl. Acad. Sci. U.S.A. 105, 11613-11618. doi:10.1073/pnas. 0801763105

Gröschel, A. H., Walther, A., Löbling, T. I., Schacher, F. H., Schmalz, H., and Müller, A. H. (2013). Guided hierarchical co-assembly of soft patchy nanoparticles. Nature 503, 247-251. doi:10.1038/nature12610

Kaiser, A. B. (2001). Electronic transport properties of conducting polymers and carbon nanotubes. Rep. Prog. Phys. 64, 1-49. doi:10.1088/0034-4885/64/1/201

Katsuki, M., Drummond, D. R., and Cross, R. A. (2014). Ectopic A-lattice seams destabilize microtubules. Nat. Commun. 5, 3094. doi:10.1038/ ncomms 4094

Koltover, I., Salditt, T., Radler, J. O., and Safinya, C. R. (1998). An inverted hexagonal phase of cationic liposome-DNA complexes related to DNA release and delivery. Science 281, 78-81. doi:10.1126/science.281.5373.78

Malcos, J. L., and Hancock, W. O. (2011). Engineering tubulin: microtubule functionalization approaches for nanoscale device applications. Appl. Microbiol. Biotechnol. 90, 1-10. doi:10.1007/s00253-011-3140-7

Needleman, D. J., Ojeda-Lopez, M. A., Raviv, U., Miller, H. P., Wilson, L., and Safinya, C. R. (2004). Higher-order assembly of microtubules by counterions: from hexagonal bundles to living necklaces. Proc. Natl. Acad. Sci. U.S.A. 101, 16099-16103. doi:10.1073/pnas.0406076101

Nogales, E., Wang, H. W., and Niederstrasser, H. (2003). Tubulin rings: which way do they curve. Curr. Opin. Struct. Biol. 13, 256-261. doi:10.1016/ S0959-440X(03)00029-0

Ojeda-Lopez, M. A., Needleman, D. J., Song, C., Ginsburg, A., Kohl, P. A., Li, Y., et al. (2014). Transformation of taxol-stabilized microtubules into inverted tubulin tubules triggered by a tubulin conformation switch. Nat. Mater. 13, 195-203. doi:10.1038/nmat3858

Raviv, U., Needleman, D. J., Li, Y., Miller, H. P., Wilson, L., and Safinya, C. R. (2005). Cationic liposome-microtubule complexes: pathways to the formation of twostate lipid-protein nanotubes with open or closed ends. Proc. Natl. Acad. Sci. U.S.A. 102, 11167-11172. doi:10.1073/pnas.0502183102

Safinya, C. R., Raviv, U., Needleman, D. J., Zidovska, A., Choi, M. C., Ojeda-Lopez, M. A., et al. (2011). Nanoscale assembly in biological systems: from neuronal cytoskeletal proteins to curvature stabilizing lipids. Adv. Mater. 23, 2260-2270. doi:10.1002/adma.201004647

Schroeder, V., Korten, T., Linke, H., Diez, S., and Maximov, I. (2013). Dynamic guiding of motor driven microtubules on electrically heated, smart polymer tracks. Nano Lett. 13, 3434-3438. doi:10.1021/nl402004s

Thess, A., Lee, R., Nikolaev, P., Dai, H., Petit, P., Robert, J., et al. (1996). Crystalline ropes of metallic carbon nanotubes. Science 273, 483-487. doi:10.1126/ science. 273.5274 .483

Conflict of Interest Statement: The author declares that the research was conducted in the absence of any commercial or financial relationships that could be construed as a potential conflict of interest.

Copyright (c) 2016 Poojari. This is an open-access article distributed under the terms of the Creative Commons Attribution License (CC BY). The use, distribution or reproduction in other forums is permitted, provided the original author(s) or licensor are credited and that the original publication in this journal is cited, in accordance with accepted academic practice. No use, distribution or reproduction is permitted which does not comply with these terms. 